Nota técnica / Technical note

\title{
Análisis de esfuerzos en pavimentos de concreto tipo whitetopping: caso de estudio vía Biblián-Zhud
}

\author{
Stress analysis in whitetopping concrete pavements: Case study the Biblián-Zhud highway
}

\author{
Jaime Bojorque-Iñeguez \\ Profesor Facultad de Ingeniería, Departamento de Ingeniería Civil, Universidad de Cuenca, Av. 12 de Abril y Agustín \\ Cuenca, Cuenca, Ecuador, 01.01.168. \\ Autor de correspondencia: jaime.bojorque@ucuenca.edu.ec \\ Fecha de recepción: 19 de octubre de 2021 - Fecha de aceptación: 1 de noviembre de 2021
}

\section{RESUMEN}

En este documento se presenta los resultados del análisis retrospectivo para determinar las posibles causas del deterioro prematuro del pavimento rígido de la vía Biblián-Zhud, arteria principal que conecta la zona austral con el norte del país. En base a las características de la vía y cargas de tráfico se realiza un análisis de los esfuerzos que se generan en las losas de pavimento. Los esfuerzos son determinados a través de ecuaciones analíticas y métodos de elementos finitos. Se estableció que los resultados por ambos métodos son muy similares. Mediante el empleo de elementos finitos se analizó, adicionalmente, el efecto de las variaciones en las cargas y del espesor del pavimento en los esfuerzos. Se determinó que bajo las cargas actuales y por las variaciones del espesor, el pavimento rígido de la vía no soporta los esfuerzos a los que está sometido, identificándose claramente el desarrollo de diferentes mecanismos de falla siendo los más concurrentes las grietas de esquina, las grietas transversales y las longitudinales.

Palabras clave: Pavimento rígido, deterioro, refuerzo delgado, esfuerzos.

\begin{abstract}
This article presents the results of a retrospective analysis to explain the probable causes of the premature deterioration of the rigid pavement of the Biblián-Zhud highway, main artery connecting the southern with the northern zone of the country. Based on the characteristics of the road and traffic loads, an analysis of the stresses generated in the pavement slabs is conducted. Stresses are determined through analytical equations and finite element methods. The results by both methods are remarkably similar. In addition, by using finite elements, the effect of variations in loads and pavement thickness on stress was also analyzed. The analysis reveals that under the current loads and due to the variations in thickness, the rigid pavement of the road does not support the stresses to which it is subjected, clearly identifying the development of different failure mechanisms, the most concurrent being corner cracks, transversal and longitudinal cracks.
\end{abstract}

Keywords: Rigid pavement, deterioration, whitetopping, stresses.

\section{INTRODUCCIÓN}

Considerando la gran inversión económica que la construcción de una vía representa, es necesario mantenerla y rehabilitarla adecuadamente para garantizar su buen desempeño durante su vida útil. Garantizando adicionalmente la seguridad y confort a los usuarios. Existen varias alternativas de rehabilitación para las vías según el grado de deterioro y tipo de superficie de rodadura. Una alternativa de rehabilitación para un pavimento flexible deteriorado es el empleo de una capa de refuerzo con hormigón adherida o no adherida, a este tipo de rehabilitación se la denomina "whitetopping" (Harrington \& Fick, 2014).

El único acceso, mediante una vía interprovincial, que tiene la provincia del Azuay, Ecuador, con el norte del país es a través la vía Biblián-Zhud que cruza la provincia del Cañar. Con la finalidad de rehabilitar esta vía de pavimento flexible, en el año 2008, se consideró el empleo de una capa de rodadura constituida por losas de concreto de cemento Portland (whitetopping) diseñadas para ser colocadas sobre el pavimento flexible, con un periodo de diseño de 20 años. Sin embargo, la vía en menos de cinco años ha presentado deterioros en varias losas, causando inconvenientes a los usuarios. Un refuerzo de hormigón whitetopping sobre un pavimento de mezcla asfáltica existente, puede clasificarse por su espesor y por el tipo de enlace con la capa de asfalto subyacente (Rasmussen \& Rozycki, 2004). Los refuerzos convencionales, tienen un espesor de más de $20.5 \mathrm{~cm}$ (8 in) y son diseñados y construidos sin considerar la posible fuerza de unión entre la capa de hormigón y la capa de asfalto. El refuerzo de hormigón está destinado a corregir el deterioro estructural 
y funcional en un pavimento asfáltico existente a un costo adecuado. La superficie del hormigón tiene buena durabilidad y rendimiento a largo plazo, y disminuye el tiempo de mantenimiento y el costo del ciclo de vida del pavimento (Tarr, Sheehan, \& Ardani, 2000; Rea \& Jensen, 2005). Sin embargo, para un buen desempeño se debe tener, muy en cuenta, el espesor de la capa de refuerzo a colocar, el espaciamiento de las juntas y la calidad del pavimento asfáltico existente. No considerar adecuadamente estas características puede resultar en fallas prematuras del pavimento (Rasmussen, McCullough, Ruiz, Mack, \& Sherwood, 2002; Lin \& Wang, 2005). La American Concrete Pavement Association (ACPA, 2011), recomienda, entre otros aspectos, las siguientes acciones para asegurar un proyecto exitoso; i) fresar el pavimento asfáltico existente para eliminar o reducir las distorsiones superficiales de 50 $\mathrm{mm}$ ( 2 in) o mayores; ii) un menor espaciamiento entre las juntas para reducir los esfuerzos por alabeo $\mathrm{y}$ deformaciones; y iii) las juntas deben aserrarse tan pronto como sea posible debido a que la ventana de tiempo para aserrar es menor que en el caso de los pavimentos convencionales.

El desempeño del refuerzo de concreto es sensible a las dimensiones de la losa, que es relativamente delgada. Cuando se compara con un pavimento de concreto convencional, los pavimentos whitetopping, generalmente, deben tener un espaciado de junta más corto, especialmente los refuerzos muy delgados. El propósito de esto es tener las grietas formadas solo en las juntas (Lin \& Wang, 2005). De lo contrario, podrían producirse grietas longitudinales en el medio de la losa, debido a esfuerzos de tracción excesivos (Eacker, 2004). La regla general para el espaciamiento entre juntas en losas de whitetopping consiste en seleccionar un espaciado de 12 a 18 veces el espesor de la losa.

La mezcla de concreto para los whitetopping no es diferente de la mezcla de concreto para un pavimento convencional. La guía de diseño de ACPA (2002) recomienda que la mezcla de concreto tenga una resistencia a la compresión mínima, a los de 28 días, de $280 \mathrm{~kg} / \mathrm{cm}^{2}(4,000 \mathrm{psi})$. Las propiedades térmicas del agregado (coeficiente térmico de expansión CTE, conductividad térmica y calor específico) y la granulometría del agregado deben considerarse en el diseño de la mezcla. El CTE es de interés teniendo en cuenta que la literatura muestra que hay un aumento significativo en los esfuerzos en la capa de whitetopping debido a los gradientes térmicos (Roesler, Harvey, Farver, \& Long, 1998; Wu, \& Tia, 2007).

Para el diseño de refuerzos whitetopping los métodos generalmente empleados son el de la ACPA (2002) y AASHTO (1993). Estos métodos, para la definición del espesor de la losa, consideran el tráfico de vehículos pesados, la resistencia a la flexión del concreto y el valor de soporte de la cimentación. Para carreteras primarias y autopistas interestatales, la guía de diseño recomienda un espesor que van desde $20.5 \mathrm{~cm}$ ( 8 in) a $30.5 \mathrm{~cm}$ (12 in).

Los principales tipos de deterioros observados en los pavimentos de refuerzo tipo whitetopping son (Rasmussen \& Rozycki, 2004; Rasmussen \& Ayers, 2003; Silfwerbrand. 1997): grietas de esquina, grietas longitudinales, grietas transversales y fallas en las juntas. Causas de falla, típicas, que se producen en los pavimentos rígidos son presentadas en la Norma ASTM D6433, el Manual de Identificación de Deterioros del U.S.
Department of Transportation (Miller \& Bellinger, 2014), el Catálogo de Deterioros de Pavimentos Rígidos, DIRCAIBEA (DIRCAIBEA, 2002), entre los documentos más relevantes para el presente estudio.

Las grietas de esquina ocurren cuando se excede el límite de fatiga del material de hormigón, que depende de la relación esfuerzo-resistencia y la magnitud de las aplicaciones de carga. Esto enfatiza aún más la necesidad de un buen pavimento asfáltico subyacente como capa de soporte para el refuerzo de concreto. Las causas del deterioro de las grietas de esquina, grietas transversales y longitudinales son la repetición de las cargas pesadas del tránsito en combinación con la acción del bombeo a través de bordes y juntas, que debilita y erosiona el apoyo de la cimentación; con deficiente transmisión de cargas entre las juntas, provocando la sobrecarga. El alabeo de las losas por variaciones térmicas o de contenido de humedad, también, generan este tipo de agrietamiento. En el caso de grietas transversales y longitudinales, adicional a las causas indicadas, una inadecuada ejecución de la junta; aserrado tardío de la junta; y losas con una relación longitud/ancho excesiva, contribuyen al agrietamiento. Es importante indicar que la severidad del agrietamiento longitudinal y transversal es sensible al espesor de la losa y al tipo de terreno de cimentación.

Los pavimentos rígidos responden a las cargas de varias maneras. Las cargas generadas afectan el desempeño de la estructura tanto a corto como a largo plazo. Los tres esfuerzos principales que se generan en un pavimento rígido son (Huang, 2012): i) esfuerzos por alabeo (curling), ii) esfuerzos de contracción y expansión (warping), y iii) esfuerzos por cargas (loading). Los esfuerzos por alabeo se producen por las diferencias de temperatura entre la superficie superior e inferior de la losa de hormigón hidráulico, haciendo que la losa se curve. Dado que el peso de las losas y el contacto con la base restringen su movimiento, se generan esfuerzos. Durante condiciones de clima cálido (por lo general, durante el día), la parte superior de la losa puede estar más caliente que la inferior, lo que produce un curvado en la dirección opuesta. Durante un clima más frío (por ejemplo, de noche o cuando se produce un frente frío), la superficie se enfría más rápidamente que la parte inferior, haciendo que la parte superior de la losa se contraiga más rápido que la inferior, lo que hace que la losa se curve en los bordes. Además del alabeo, las temperaturas ambientales y humedades hacen que las losas de cemento se expandan, cuando están calientes y se contraigan cuando están frías, lo que causa el movimiento en las juntas, fenómeno conocido como deformación por humedad (warping). Sin embargo, estos esfuerzos son muy pequeños en comparación con los generados por cambios de temperatura y/o tráfico, por lo que generalmente no se los determina (Huang, 2012).

Los esfuerzos generados por las cargas del tráfico, en general, son los mayores en el diseño de las vías. Estas cargas provocan esfuerzos de compresión y de tracción dentro de la losa y cualquier otra losa adyacente, cuando exista transferencia de carga entre losas. A lo largo de las juntas, los bordes del pavimento tienden a curvarse hacia arriba cuando la superficie del concreto es más seca y fría que la inferior. Los bordes curvados se convierten en voladizos, lo que genera que el tráfico que pasa sobre las juntas causa una desviación vertical repetitiva que crea agrietamiento por fatiga en el hormigón. Los cambios de esfuerzos que se generan deben ser compensados por la 
resistencia del hormigón. Dado que el concreto de cemento Portland es mucho más resistente en compresión que en tracción, los esfuerzos por tracción tienden a controlar el diseño del pavimento. En general, para la determinación de los esfuerzos se cuenta con ecuaciones analíticas y programas numéricos. Las ecuaciones empleadas para estimar los esfuerzos de deformación fueron desarrolladas por Westergaard (Huang, 2012), adicionalmente se tienen programas computacionales en base a elementos finitos como EverFE (Davids et al., 2003).

El presente estudio se centra en establecer las posibles causas del deterioro prematuro del pavimento tipo whitetopping de la vía Biblián-Zhud, ello en base a un extenso análisis de esfuerzos generados por las cargas de tráfico y cambios de temperatura. Se emplean tanto ecuaciones analíticas como métodos numéricos para realizar una comparación entre ambos métodos y seleccionar el óptimo para analizar factores adicionales como el incremento de cargas y la reducción de espesor de las losas.

\section{MATERIALES Y MÉTODOS}

\subsection{Zona de estudio y características de la vía}

La vía Biblián-Zhud tiene una longitud aproximada de 53 $\mathrm{km}$, de los cuales $36 \mathrm{~km}$, en tres tramos: Zhud $(0+000)$ El Juncal $(9+124)$; Cañar $(24+609)$ - Campo Alegre (46+069); y Campo Alegre (47+509) - Biblián (53+019), tienen una capa de rodadura con pavimento rígido tipo whitetopping y los restantes con pavimento flexible. En la Fig. 1 se presenta la ubicación de la vía dentro de Ecuador. Las losas para la capa de rodadura fueron diseñadas en 22 $\mathrm{cm}$ de espesor con el empleo de pasadores en las juntas transversales y varillas de unión en las longitudinales. La calzada de vía es de dos carriles, uno en cada dirección, con espaldones. Las losas de concreto tienen una longitud de $4.5 \mathrm{~m}$ por $4.0 \mathrm{~m}$ de ancho, por carril, empleándose pasadores de $25 \mathrm{~mm}$ en las juntas transversales. Las losas fueron diseñadas con una resistencia a la flexión (módulo de rotura) de $4.41 \mathrm{MPa}\left(45 \mathrm{~kg} / \mathrm{cm}^{2}\right)$. Actualmente, a lo largo de toda la vía se observan varios deterioros, especialmente, grietas de esquina, grietas longitudinales y grietas transversales. Como hipótesis de partida se considera que el espesor de la losa, las cargas de los vehículos y la resistencia de los materiales contribuyeron al deterioro prematuro del pavimento rígido. Vale la pena mencionar que, factores adicionales como el clima y el bombeo (lavado de finos) aceleran los procesos de deterioro del pavimento. Estos factores deberían considerarse si se requiere entender la evolución en el tiempo del proceso de deterioro, lo que va fuera del alcance del presente estudio.

Los parámetros de partida para el análisis de esfuerzos corresponden a los siguientes: espesor de la losa $22 \mathrm{~cm}$, coeficiente de Poisson de 0.15, módulo de elasticidad del concreto 3'725,000 psi (para un f'c $=360 \mathrm{~kg} / \mathrm{cm}^{2}$ ), coeficiente térmico del concreto $6 \times 10^{-6 \circ} \mathrm{F}$. El módulo de reacción del soporte es de 209 pci; este valor fue determinado en base a ensayos de deflectometría FWD. Las cargas consideradas son las máximas estipuladas para buses y camiones según el Ministerio de Transportes y Obras Públicas del Ecuador MTOP (Tabla Nacional de Pesos y Dimensiones). Para eje simple rueda doble es de 11 ton $(108 \mathrm{kN})$ y para eje tándem es de 20 ton $(196 \mathrm{kN})$. Para la determinación del diferencial de temperatura en la losa, se consideró el registro de temperatura ambiente en la estación Cañar del 2012 (Estación Cañar M0031, INAMHI, 2015) en donde la máxima temperatura llega a los $29^{\circ} \mathrm{C}$ (febrero) y la mínima a $-2^{\circ} \mathrm{C}$ (diciembre). Considerando estas temperaturas ambientales, se asume una diferencia de temperatura en las losas de $15^{\circ} \mathrm{C}$.

\subsection{Esfuerzos en las losas}

Los cálculos mediante ecuaciones analíticas representan una aproximación de los esfuerzos que se esperan en los pavimentos, con fines de diseño. Programas más avanzados como los basados en elementos finitos proporcionan información adicional que es de utilidad para el análisis del comportamiento del pavimento. El paquete computacional EverFE proporciona una
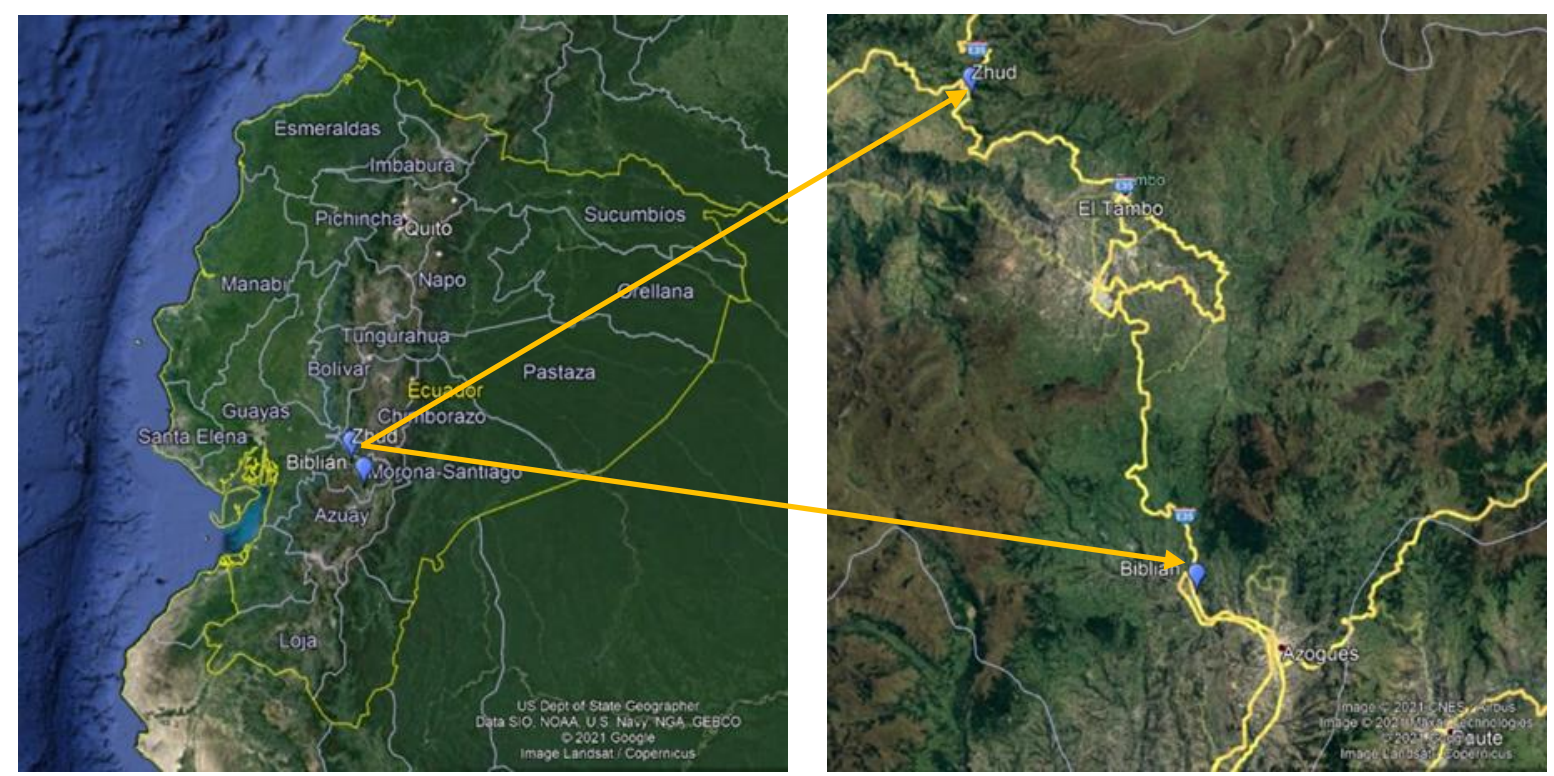

Figura 1. Ubicación geográfica de la vía Biblián-Zhud en la provincia del Cañar, Ecuador. 
plataforma simple y fácil de usar para el análisis de esfuerzos de pavimento rígidos de configuraciones variables y en diferentes condiciones de carga, incluidas cargas térmicas inducidas por gradientes de temperatura. Detalles más específicos del programa de elementos finitos EverFe, empleado en este estudio, se pueden obtener en Davids (2003). Maske et al. (2013) analizó el diseño de pavimentos rígidos mediante métodos empíricos y métodos basados en elementos finitos para comparar los resultados de los esfuerzos del pavimento. Las conclusiones extraídas del estudio son que los métodos de elementos finitos pueden proporcionar un diseño óptimo y económico. Adicionalmente, los métodos de elementos finitos proporcionan una estimación más precisa del comportamiento de las secciones críticas del pavimento rígido bajo cargas aplicadas.

Debido a la dificultad de contar con datos adecuados de la disposición de los pasadores y su desempeño en la estructura, y al no contar con las características geomecánicas de los materiales capa por capa, se realiza el análisis con un modelo de losa simple asentada sobre una estructura que comprende la cimentación. Adicionalmente, el modelo propuesto concuerda muy bien con los resultados de las ecuaciones analíticas, validándose el empleo del mismo. Para todos los análisis, cada losa fue dividida en una retícula de $32 \times 32 \times 4$ elementos (en sus ejes X, Y y Z, respectivamente). Esta retícula permite una buena aproximación en el cálculo, a la vez que la capacidad computacional requerida y el tiempo de cada corrida permanecen a niveles razonables.

Se evalúan las cargas considerando, primero, un eje simple de neumático doble y luego el efecto de un eje tándem. Con la configuración empleada se verifican los esfuerzos en el interior, en la esquina y en los bordes de la losa. En el caso de los gradientes positivos (parte superior más caliente que la inferior), las cargas de tránsito son aplicadas en el borde de las losas, produciéndose esfuerzos máximos en la cara inferior de la losa. En el caso de los gradientes negativos (parte superior más fría que la inferior), las cargas de tránsito son aplicadas en la esquina de las losas, produciéndose esfuerzos máximos en la cara superior.

\section{RESULTADOS Y DISCUSIONES}

En base a los parámetros indicados, ecuaciones analíticas y métodos de elementos finitos, se evalúa el efecto de los esfuerzos en el interior, borde y esquina de las losas. Los resultados de la acción combinada de los esfuerzos por temperatura y carga vehicular, según las ecuaciones analíticas dan que el esfuerzo en el interior es de 3.17 $\mathrm{MPa}$, en el borde $4.38 \mathrm{MPa}$, y en la esquina $2.52 \mathrm{MPa}$. El esfuerzo combinado en el borde de la losa representa el más crítico equivalente al $99 \%$ de la resistencia de diseño del concreto de $4.41 \mathrm{MPa}$. Un aumento en la carga provocaría que se sobrepase la resistencia del concreto generando fallas en la losa. Así también, una disminución en la resistencia del hormigón iniciaría de igual manera el fallo.

\subsection{Resultados de esfuerzos en la losa por elementos finitos}

En la Fig. 2 se proporciona los resultados generados en EverFE, donde se presentan los esfuerzos en los bordes, centro y esquina de la losa para la carga de eje simple de 11 ton. El esfuerzo que se produce en el borde longitudinal (Fig. 2a) es de $4.38 \mathrm{MPa}$, lo que corresponde al $99 \%$ de la resistencia del material. Cualquier cambio en el espesor de la losa, resistencia del material o carga superior a la establecida, produciría el agrietamiento y falla de la losa. En la Fig. 2b se muestra el análisis para esfuerzos en la esquina y borde lateral, para la carga de 11 ton y gradiente térmico positivo. En este caso, los esfuerzos máximos se producen en el borde transversal de la losa y en la parte inferior de la misma, alcanzando un valor de $2.60 \mathrm{MPa}$, es decir, un 59\% de la resistencia del concreto. Para el caso de los esfuerzos en la esquina y gradiente térmico negativo, estos esfuerzos se producen en la parte superior de la losa cerca de la esquina con un valor de $3.02 \mathrm{MPa}$, siendo el $68 \%$ de la resistencia del material, los esfuerzos generados en esta zona son los que generan grietas de esquina.

La comparación de resultados entre las ecuaciones analíticas y el programa de elementos finitos se incluyen en la Tabla 1. Se puede apreciar que los parámetros ingresados en el programa representan adecuadamente los resultados analíticos, validándose el empleo del programa EverFE. Esta validación sirve para posteriores análisis de los parámetros de la estructura del pavimento y cargas con ejes tándem y gradientes térmicos positivos y negativos.

Tabla 1. Esfuerzos mediante ecuaciones analíticas y EverFE.

\begin{tabular}{lcc}
\hline Esfuerzo (MPa) & $\begin{array}{c}\text { Métodos } \\
\text { analíticos }\end{array}$ & EverFE \\
\hline En el interior & 3.17 & 3.28 \\
En el borde & 4.38 & 4.38 \\
En la esquina & 2.52 & 3.02 \\
\hline
\end{tabular}

Como se indicó anteriormente, los esfuerzos generados en el borde de la losa están muy cercanos a los valores de diseño. Para la carga de 11 ton en un eje simple neumático doble, espesor de $22 \mathrm{~cm}$ y resistencia del concreto a la flexión de 4.41 $\mathrm{MPa}$, las losas están a límite de su resistencia, pero deberían funcionar según el análisis de esfuerzos. Sin embargo, un incremento en las cargas del tráfico o una disminución en resistencia del concreto o en el espesor del pavimento, provocaría el deterioro del pavimento.

Un incremento de $20 \%$ de la carga prevista de 11 ton (factor 1.2 recomendado por la PCA, 1984), a 13.2 ton, provoca que el esfuerzo llegue a los $4.71 \mathrm{MPa}$ valor superior al de diseño de $4.41 \mathrm{MPa}$. En la Fig. 3a se incluyen los esfuerzos generados con un aumento de carga a 13.2 ton y en la Fig. $3 \mathrm{~b}$ se indica una imagen del tipo de deterioro encontrado en la vía en el kilómetro 48+800. De la Fig. 3 se observa que la zona de esfuerzos mayores se produce en el borde y centro de la losa lo que puede provocar la aparición y generación de grietas transversales y el deterioro del pavimento, como se observa in situ en algunos sitios de la vía, por ejemplo, en la abscisa 48+800.

En la Fig. 4 se puede apreciar el mecanismo que se desarrolla en la generación de grietas de esquinas. Si bien, los esfuerzos no llegan a límite de la resistencia, se puede 


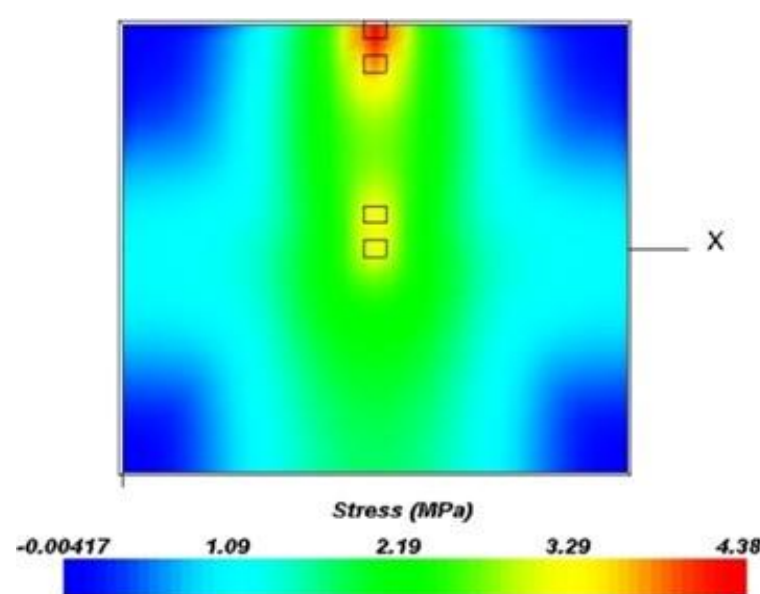

a) Esfuerzos en la losa, máximo 4.38 MPa borde, 3.28 MPa interior centro

Figura 2. Esfuerzos en: a) bordes y centro; b) esquina, carga 11 ton

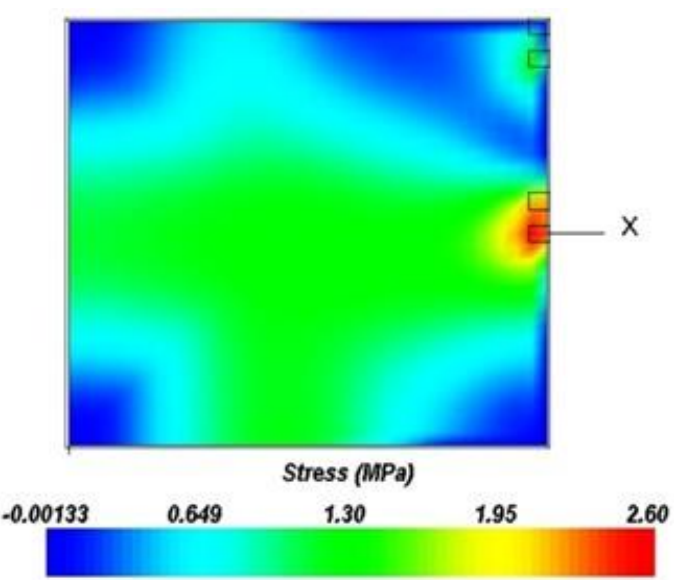

b) Esfuerzos en la losa, máximo 2.60 MPa en el borde.

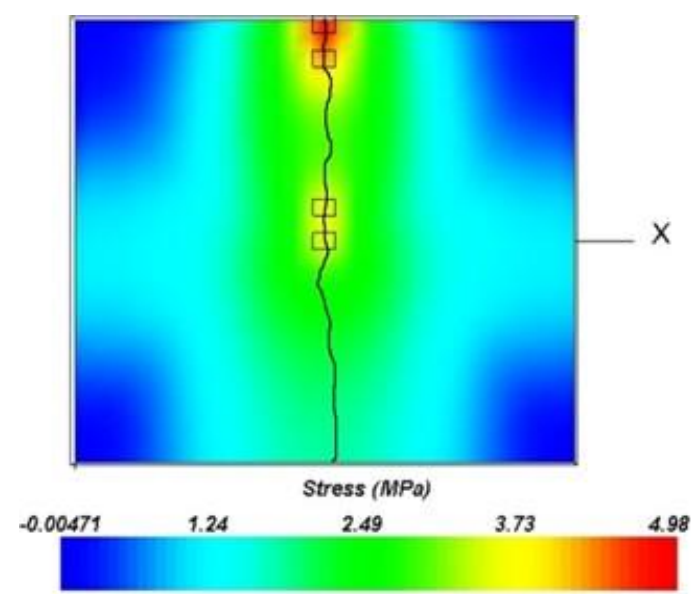

a) Esfuerzos por Elementos Finitos, zonas de esfuerzos máximos

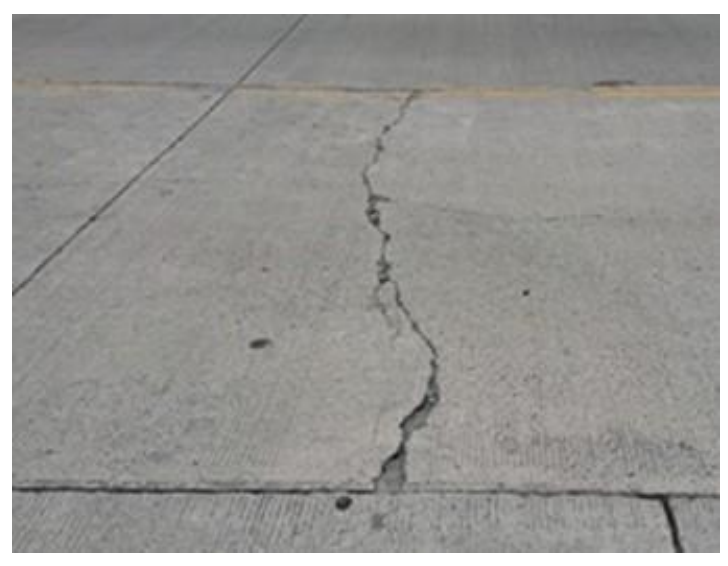

b) Agrietamiento en la vía abscisa $48+800$

Figura 3. Esfuerzos en el borde de la losa para carga de 13.2 ton.

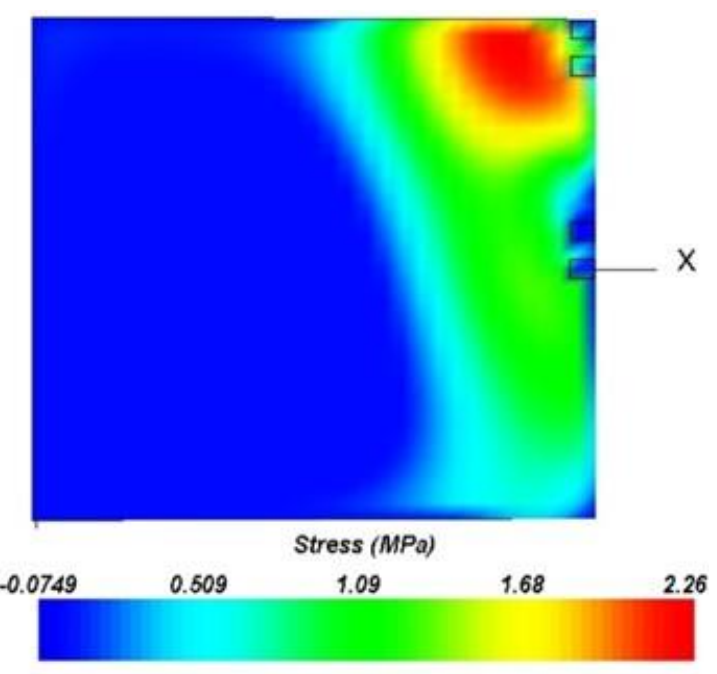

a) Esfuerzos por Elementos Finitos, zonas de esfuerzos máximos

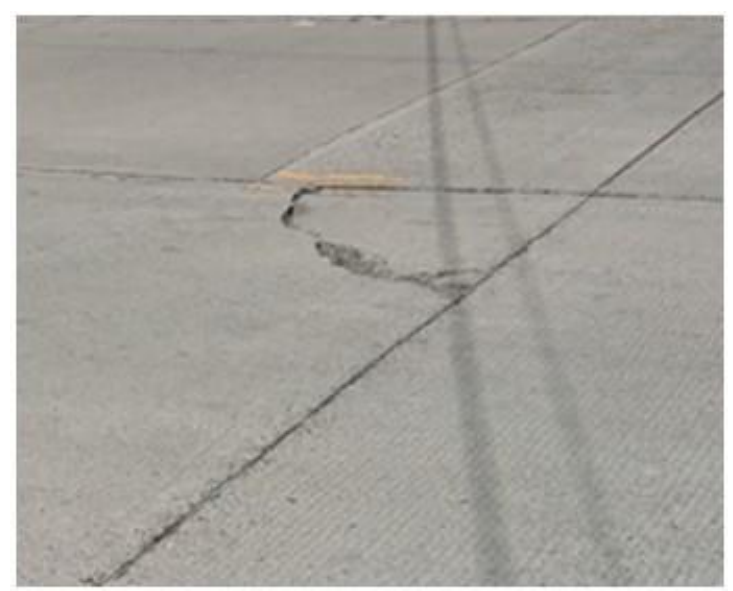

b) Agrietamiento en la vía abscisa $50+766$

Figura 4. Esfuerzos en la esquina de la losa, carga 13.2 ton. 


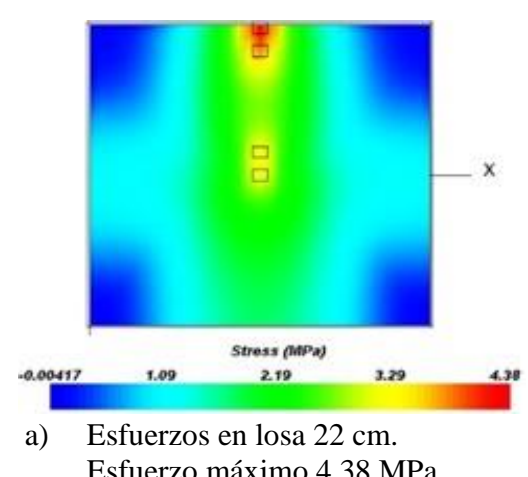

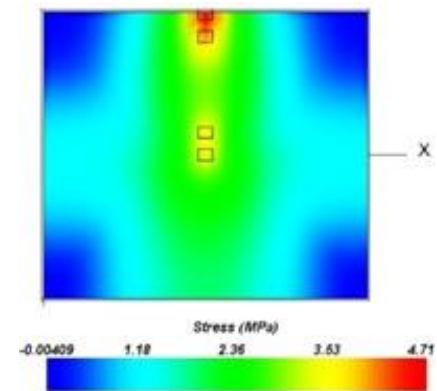

b) Esfuerzos en losa $21 \mathrm{~cm}$. Esfuerzo máximo 4.71 MPa

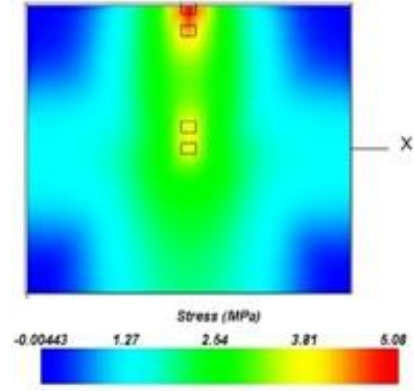

c) Esfuerzos en losa $20 \mathrm{~cm}$. Esfuerzo máximo $5.08 \mathrm{MPa}$

Figura 5. Efecto del espesor de losa en esfuerzos en el borde.

observar la concentración de esfuerzos. Las grietas de esquina son deterioros frecuentemente encontrados a lo largo de la vía Biblián-Zhud (ejemplo Fig. 4b), indicando con ello que se ha excedido el límite de fatiga del hormigón.

En la Fig. 5 se presenta de forma gráfica el efecto de la disminución del espesor del pavimento en los esfuerzos en el borde de la losa. En base a la extracción de núcleos, se determinó que algunas losas tienen espesores menores a los $22 \mathrm{~cm}$, de aquí que se consideró el análisis de otros espesores para analizar el efecto de la disminución del mismo en los esfuerzos. En caso de la losa de $22 \mathrm{~cm}$, el esfuerzo máximo está al límite de la resistencia (Fig. 5a), para una losa de $21 \mathrm{~cm}$, el esfuerzo de $4.71 \mathrm{MPa}$ sobrepasa la resistencia del material (Fig. 5b), y para una losa de 20 $\mathrm{cm}$, el esfuerzo máximo que se genera es de $5.08 \mathrm{MPa}$ sobrepasando la resistencia de diseño del concreto en un $15 \%$ (Fig. 5c).

De la Fig. 5 se observa que hay una concentración de esfuerzos en la parte central de la losa lo que generaría el inicio de grietas transversales. Al considerar la carga de 11 ton actuante en la esquina, los esfuerzos debido al cambio de espesor no aumentan en mayor medida, para una losa de $22 \mathrm{~cm}$, el esfuerzo en la esquina es de 3.02 $\mathrm{MPa}$, para $21 \mathrm{~cm}$ de $3.16 \mathrm{MPa}$ y para $20 \mathrm{~cm}$ de $3.31 \mathrm{MPa}$. $\mathrm{Al}$ aumentar la carga, a 13.2 ton, el esfuerzo máximo que se genera en la losa de $20 \mathrm{~cm}$ es de $3.72 \mathrm{MPa}$ lo que equivale al $84 \%$ de la resistencia del concreto. Aquí se aprecia la generación de esfuerzos que pueden conducir a la formación de grietas de esquina. Para que se sobrepase la resistencia del hormigón, la carga del eje debe ser del orden de 17.5 ton.

Al considerar el efecto de un eje tándem, los esfuerzos no son tan altos como en el eje simple, sin embargo, estos camiones con frecuencia llevan sobrepeso. Aunque los esfuerzos no superan a la resistencia del concreto, se presenta una concentración de esfuerzos que pueden generar grietas transversales y/o longitudinales en el caso de un incremento en las cargas del tráfico o una disminución en resistencia del concreto o en el espesor del pavimento, o una combinación de éstas. Al analizar los esfuerzos que se generan en el borde transversal de la losa por eje tándem se aprecia el desarrollo de una zona de concentración de esfuerzos que pueden generar el desarrollo del agrietamiento longitudinal, iniciando en la junta y prolongándose longitudinalmente.

Considerando que el aumento de la carga por eje afecta directamente a los esfuerzos en la losa, durante el mes de febrero 2018, el MTOP realizó el conteo y pesaje de los vehículos que circulan en la vía Biblián-Zhud, sector San
José de Inganilla. En el Informe de Tráfico (MTOP, 2018) se incluye el reporte de pesaje de cada eje según el tipo de vehículo. Los tipos de vehículos considerados en el reporte, en los cuales se realizó el pesaje, son: 2D, 2DA, 2DB, V2DB, 3A, V3A, 3S2 y 3S3 (Tabla Nacional de Pesos y Dimensiones). Las cargas medidas y máximas permitidas en los ejes de los diferentes vehículos se presentan en la Tabla 2.

Tabla 2. Cargas medidas a través de pesaje en la vía Biblián-Zhud.

\begin{tabular}{lr}
\hline Tipo de camión & \multicolumn{1}{c}{$\begin{array}{c}\text { Carga medida en el pesaje } \\
\text { (máxima permitida) [toneladas] }\end{array}$} \\
\hline 2D & 4.72 (máx. 4.0) \\
2DA & 6.74 (máx. 7.0) \\
2DB & 19.56 (máx. 11.0) \\
V2DB & 13.29 (máx. 11.0) \\
3A & 30.72 (máx. 20.0) \\
V3A & 30.46 (máx. 20.0) \\
3S2 & 27.83 (máx. 20.0) \\
$3 S 3$ & 30.96 (máx. tándem 20.0) \\
\hline
\end{tabular}

Se observa que, en casi todos los casos, excepto para el tipo 2DA, los pesos medidos sobrepasan los estipulados por el MTOP. Los camiones 2DB (eje simple neumático doble) y $3 \mathrm{~A}$ (eje tándem), se los toma como referencia para el análisis de esfuerzos, al ser los más críticos en cuanto a la carga. La carga del camión 2DB de 19.56 ton es $77.8 \%$ más que la permitida. Para el camión 3A (30.46 ton) la carga es $52.3 \%$ mayor.

Los esfuerzos generados por la carga de 19.56 ton, en el bode y centro de la losa, sobrepasan la resistencia del concreto lo que produciría la falla del pavimento. En el borde de la losa se generan esfuerzos del orden de 6.38 $\mathrm{MPa}$, lo que corresponde a $145 \%$ de la resistencia de diseño de 4.41 MPa. Los esfuerzos en la esquina y borde de la losa también son importantes, llegando a valores iguales que la resistencia del pavimento, cualquier cambio en resistencia y/o espesor, provocaría la falla de esquina. Estas cargas que superan los valores estipulados por el MTOP, en cuanto a pesos máximos, generan esfuerzos de fatiga en el pavimento, sin embargo, debido a otros aspectos como la calidad de la subrasante, la calidad de la estructura del pavimento, la trabazón del agregado o transferencia de carga en los pasadores, aún no han fallado todas las losas. 
En el caso del vehículo tipo 3A, al igual que el vehículo 2DB, los esfuerzos en los bordes superan la resistencia del pavimento lo que provocaría la formación de grietas transversales y longitudinales. Si bien, en la esquina los esfuerzos aún no superan la resistencia del concreto, estos representan el $84 \%$ de la resistencia de diseño. Se puede indicar que los camiones que transitan por la vía BibliánZhud están provocando grandes esfuerzos que superan la resistencia del pavimento, generando el deterioro del pavimento en ciertas zonas.

\section{CONCLUSIONES}

En la actualidad, la vía Biblián-Zhud presenta diferentes fallas como grietas de esquina, grietas longitudinales y grietas transversales, entre las más frecuentes y severas. Los esfuerzos generados en las losas debido a los cambios térmicos y cargas de los camiones han provocado el fallo de algunas losas de la vía. En base al presente estudio se puede indicar que el deterioro prematuro del pavimento rígido tipo whitetopping de la vía Biblián-Zhud no es atribuible a una sola causa, sino a la acción de varios factores, entre los que constan las cargas de tráfico que sobrepasan a las de diseño, la variación en el espesor de la losa y la resistencia del concreto.

Se ha identificado que existen tramos en condiciones de excelentes a buenos que, debido a otros aspectos, que no son posibles valorar en la actualidad, como la calidad de la estructura del pavimento asfáltico al momento de la construcción y la transferencia de carga en los pasadores, entre otras, aún mantienen un desempeño adecuado. La heterogeneidad de la estructura actual hace que el desempeño del pavimento losa por losa sea variable. El deterioro en el carril derecho en el tramo Zhud-Juncal es más severo que en el resto de la vía. Esto podría deberse a que los camiones van desde las provincias del Guayas y Chimborazo hacia Cañar con mayores cargas de mercadería.

De los análisis de esfuerzos realizados se observó que, para cargas mayores a las de diseño, los esfuerzos en el borde y centro de la losa sobrepasan la resistencia del concreto lo que produce el agrietamiento del pavimento. Empleando los pesos de los camiones medidos in situ, en el borde de la losa se generan esfuerzos del orden de 6.38 $\mathrm{MPa}$, lo que corresponde a $145 \%$ de la resistencia de diseño de 4.41 MPa. Los esfuerzos en la esquina de la losa también son significativos, representando el $100 \%$ de la resistencia del pavimento. Estas cargas superan los valores estipulados por el MTOP, en cuanto a pesos máximos, generando esfuerzos de fatiga en el pavimento, lo que provoca el fallo de las losas.

Para prevenir el deterioro acelerado de la vía es fundamental realizar un control de los pesos máximos de los camiones que circulan por la carretera, debiendo cumplirse lo estipulado por el MTOP. Es necesario realizar una rehabilitación a la vía, considerado un diseño adecuado que englobe el desempeño de la estructura actual. Así también, es conveniente para proyectos futuros, en la zona o en otras del país, realizar estudios de tráfico y proyecciones adecuadas para estimar correctamente las tasas de crecimiento del parque automotor, en especial de los camiones al ser los más agresivos referente a la generación de esfuerzos en la vía.

\section{AGRADECIMIENTO}

El autor desea agradecer al Ministerio de Transporte y Obras Públicas del Cañar y a la Empresa Pública de la Universidad de Cuenca (UCuencaEP), entidad contratante y contratista, respectivamente, del estudio de la vía ZhudBiblián.

\section{REFERENCIAS}

AASHTO. (1993). AASHTO Guide for design of pavement structures. American Association of State and Highway Transportation Officials, Washington, DC, U.S.

ACPA. (2002). Portland cement concrete overlays: State of the technology synthesis. American Concrete Pavement Association. Product Code SP045P. Skokie, IL, U.S.

ACPA. (2011). AirPave guide ACPA. American Concrete Pavement Association, 9450 Bryn Mawr, Suite 150 Rosemont, IL 60018, U.S.

ASTM D6433. (2007). Standard practice for roads and parking lots pavement condition index surveys. ASTM International, West Conshohocken, PA, www.astm.org.

Davids, B. (2003). EverFE theory manual. University of Maine, Civil Engineering Department, U.S.

Davids, W. G., Wang, Z. M., Turkiyyah, G., Mahoney, J., \& Bush, D. (2003). Finite element analysis of jointed plain concrete pavement with EVERFE 2.2. Journal of the Transportation Research Board, National Research Council, US, 92-99.

DIRCAIBEA. (2002). M5.2. Catálogo de deterioros de pavimentos rígidos. Reporte técnico. Consejo de Directores de Carreteras de Iberia e Iberoamérica.

Eacker, M. (2004). Three year evaluation of whitetopping projects on M-46. Michigan Department of Transportation, U.S.

Harrington, D., Fick, G. (2014). Guide to concrete overlays, sustainable solutions for resurfacing and rehabilitating existing pavements. 3ra ed. American Concrete Pavement Association, ACPA.

Huang, Y. (2012) Pavement analysis and design. Pearson/Prentice Hall, 2da edición.

INAMHI. (2015). Anuario meteorológico, Nro. 52-2012. Instituto Nacional de Meteorología e Hidrología, Quito, Ecuador.

Maske, N. A., Anandkumar, A., \& Majumder, A. (2013). Analysis of rigid pavement stresses by finite element method \& Westergaard's method by varying sub-grade soil properties. International Journal of Engineering Science Invention, 2(3), 52-55.

Miller, J., \& Bellinger, W. (2014). Distress identification manual for the long-term pavement performance program. U.S. Department of Transportation - Federal Highway Administration. Publication NO. FHWAHRT-13-092.

MTOP. (2018). Informe de tráfico en la vía ZhudBiblián, sector San José de Inganilla. Reporte técnico. Ministerio de Transporte y Obras Públicas, Ecuador. 
Lin, D. F., \& Wang, H. Y. (2005). Forensic investigation of ultra-thin whitetopping failures in Taiwan. ASCE Journal of Performance of Constructed Facilities, 19(2).

PCA. (1984). Thickness design for concrete highway and street pavements. Portland Cement Association, U.S.

Rasmussen, R. O., McCullough, B., Ruiz, J., Mack, J., \& Sherwood, J. (2002). Identification of pavement failure mechanisms at FHWA accelerated loading facility ultrathin whitetopping project. Transportation Research Record, 1816(1), 148-155. htpps://doi.org/10.3141/1816-16

Rasmussen, R. O., \& Ayers, M. (2003). Performance and design of whitetopping overlays for heavily trafficked pavements. Final Report for Concrete Pavement Technology Program Tas 3(99). Federal Highway Administration, Washington, D.C., U.S.

Rasmussen, R. O., \& Rozycki, D. K. (2004). Thin and ultra-thin whitetopping - A synthesis of highway practice. NCHRP Synthesis 338, Transportation Research Board, Washington, D.C., U.S.
Rea, R. C., \& Jensen, W. G. (2005). A concrete overlay on an asphalt road. The International Journal of Pavement Engineering, 6(2), 111-114. htpps://doi.org/10.1080/10298430500068670

Roesler, J., Harvey, J., Farver, J., \& Long, F. (1998). Investigation of design and construction issues for long life concrete pavement strategies. Draft report for the California Department of Transportation, Institute of Transportation Studies, Univ. of California, Berkeley, U.S.

Silfwerbrand, J. (1997). Whitetoppings - Swedish field test and recommendations. Proc., 6th Int. Purdue Conf. on Concrete Pavement, Design and Materials for High Performance, Vol. II 234-244.

Tarr, S. M., Sheehan, M., \& Ardani, A. (2000).

Mechanistic design of thin whitetopping pavements in Colorado. Transportation Research Record, 1730(1), 64-72. https://doi.org/10.3141/1730-08

Wu, C. L., Tia, M., \& Choubane, B. (2007). Forensic investigation of ultrathin whitetopping pavements in Florida. ASCE Journal of Performance of Constructed Facilities, 21(1). 